\title{
DEFECOGRAFÍA POR RESONANCIA MAGNÉTICA
}

Dr. Andrés O'Brien S.

Sección de Abdomen, Diagnóstico por Imágenes. Clínica Las Condes, Santiago-Chile.

\section{MAGNETIC RESONANCE DEFECOGRAPHY}

Abstract: The term disorders of the anorectal function encompasses a group of diseases commonly present in gastroenterological consultation; conditions that have a significant impact on patients' quality of life. Imaging studies, mainly defecography, are essential for the diagnosis of these diseases. Taking into account that there is overlap of several of them in the same patient, some may be underdiagnosed when relying solely on anamnesis and physical examination. Given the multiplanar capability of $M R I$, visualization of perirectal soft tissue, its lowest invasiveness in relation with other studies and the absence of ionizing radiation or barium, MRI defecography has been installed as an imaging modality widely used in our milieu, replacing the classical defecography performed under fluoroscopy.

Keywords: Defecography, Constipation,MRI.

Resumen: Los trastornos de la función anorrectal están constituídos por un grupo de patologías frecuentes en la consulta gastroenterológica, que determinan un impacto en la calidad de vida. Los estudios de imagen, en especial la defecografía, son fundamentales para el diagnóstico de estas patologías; si se considera que hay traslape de varias de ellas en un mismo paciente, algunas pueden quedar subdiagnosticadas al contar solamente con la anamnesis y el examen físico. Dada la capacidad multiplanar de la resonancia magnética, la visualización de las partes blandas perirrectales, su menor invasividad en relación a otros estudios y el no uso de radiación ionizante ni bario, la defecografía por resonancia magnética se ha instalado como una modalidad de imagen de amplio uso en nuestro medio,

O'Brien A. Defecografía por resonancia magnética. Rev Chil Radiol 2010; 16(1): 11-16.

Correspondencia: Dr. Andrés O’Brien S.

aobrien@clinicalascondes.cl

Trabajo recibido el 28 de diciembre de 2009, aceptado

para publicación el 26 de enero de 2010. reemplazando a la defecografía clásica efectuada bajo fluoroscopía.

Palabras clave: Defecografía, Constipación, Resonancia Magnética.

\section{Introducción}

Los trastornos de la función anorrectal constituyen aproximadamente un $10-20 \%$ de la consulta gastroenterológica ${ }^{(1,2)}$, con un significativo impacto en la calidad de vida, lo que se acentúa en pacientes geriátricos. Los motivos más frecuentes de consulta son: constipación, incontinencia, dolor y prolapso, que suelen traslaparse en grado variable. De éstos, la constipación es el síntoma más frecuente; se define por los siguientes síntomas: heces duras, evacuación menos de tres veces por semana, esfuerzo excesivo, evacuación incompleta y/o maniobra digital para facilitarla ${ }^{(3)}$. Es frecuentemente multifactorial, siendo sus posibilidades etiológicas muy amplias, incluyendo enfermedades sistémicas, secundaria a fármacos, causas estructurales colorrectales y trastornos de la motilidad colorrectal.

El diagnóstico de estas patologías es difícil; incluye la anamnesis, el examen físico, y algunos test específicos como la manometría anorrectal, estudio de tránsito colónico y de intestino delgado, colonoscopía, enema baritada de colon, endosonografía del esfínter anal y defecografía bajo fluoroscopía (DF)(4) o por resonancia magnética (DRM) $)^{(2,5)}$. El rol de las imágenes es fundamental, dado que los hallazgos del examen físico en muchas ocasiones son limitados o equívocos, llevando a un manejo inapropiado que finalmente puede derivar en más de una intervención quirúrgica.

Por décadas, la DF se ha utilizado para la evaluación de pacientes con constipación crónica o trastornos defecatorios. Sin embargo, ésta presenta varias limitaciones para su adecuada evaluación: requiere administrar contraste a la vejiga, vagina, recto e intestino delgado y además marcar el margen anal con pequeños elementos radioopacos. La visualización de las imágenes es proyeccional, es decir, corresponde a la sumatoria de muchos planos, no visualiza los tejidos 
blandos perirrectales, en especial los músculos del piso pélvico, utiliza radiación ionizante y es operador dependiente. A esto se suma que el examen puede ser muy embarazoso para algunos tipos de pacientes, dado que deben defecar en presencia del radiólogo y eventualmente de un auxiliar.

Con los nuevos avances tecnológicos, en especial nuevas secuencias rápidas de resonancia magnética (RM) que permiten evaluar dinámicamente el piso pélvico manteniendo una alta resolución espacial y por contraste, se desarrolló la DRM a fines de la pasada década. En oposición a la DF, no requiere administrar contraste a la vejiga, vagina ni asas de intestino delgado; sólo se administra gel de ultrasonido al recto. Visualiza los tejidos blandos perirrectales, tanto en fase estática como dinámica, permite obtener imágenes en uno o múltiples planos y no proyeccionales, no utiliza radiación ionizante y es menos operador dependiente; de hecho, no es necesaria la presencia del radiólogo durante el examen. Además, el paciente efectúa las maniobras defecatorias estando solo en la sala de RM.

\section{Técnica}

Hay dos tipos de magneto para realizar este examen: uno abierto de 0,5 Tesla, en el cual el paciente puede estar sentado, y otro cerrado de 1,5 Tesla, con el paciente en posición horizontal (supina). El magneto abierto es menos disponible y, dado su menor teslaje, el cuociente señal/ruido (SNR) es menor. Por otra parte, el magneto cerrado es ampliamente disponible, con mejor SNR; la principal crítica es el hecho de que el paciente no esté en una posición fisiológica. No obstante, estudios realizados no han demostrado diferencias clínicamente significativas entre DRM realizadas en los dos tipos de magneto (posición sentada versus horizontal) ${ }^{(6)}$, por lo que nos referiremos a las DRM realizadas en magneto cerrado.

La DRM consta de dos partes, iniciándose con una evaluación estática de la pelvis con el paciente en reposo. Esta se realiza con cortes ponderados en T2 Turbo spin-echo (TSE) de alta resolución (TR:3600 ms, TE: $121 \mathrm{~ms}$, espesor de corte: $4 \mathrm{~mm}$, FOV: 200 $\mathrm{mm}$, matriz: $256 \times 512$ ), en planos axial (Figura 1), coronal y sagital para un análisis detallado de la anatomía, que incluye vejiga, vagina, útero, recto, asas de íleon, anexos y músculos elevador del ano y puborrectal, estos últimos con hiposeñal en este tipo de secuencia. Este examen se realiza menos frecuentemente en hombres; en ellos se evalúa la vejiga, próstata, vesículas seminales, recto y los músculos del piso pélvico. Posteriormente, a través de cánula endorrectal se administran 200-250 cc de gel de ultrasonido (existe la alternativa de administrar puré de papas mezclado con Gadolinio) ${ }^{(7)}$ y se realiza secuencia dinámica TrueFisp, Balanced FFE o
FIESTA (dependiendo de las diferentes marcas de los equipos) sagital en línea media (TR: 4,5 ms, TE: 1,8 $\mathrm{ms}$, espesor de corte: $10 \mathrm{~mm}$, FOV: $250 \mathrm{~mm}$, matriz: $320 \times 320$ ), con el paciente en reposo, seguido de las maniobras de contracción del esfínter anal, Valsalva y posterior defecación (Figura 2). Se realizan 120 mediciones, de un segundo cada una, lo que permite una secuencia cine con adecuada resolución temporal. Además, por el tipo de secuencia utilizada, que presenta contraste tanto T1 como T2, se logra una adecuada resolución espacial.

Posteriormente, las imágenes son analizadas en una estación de trabajo, en la que pueden ser evaluadas en forma apropiada tanto las imágenes estáticas como dinámicas.

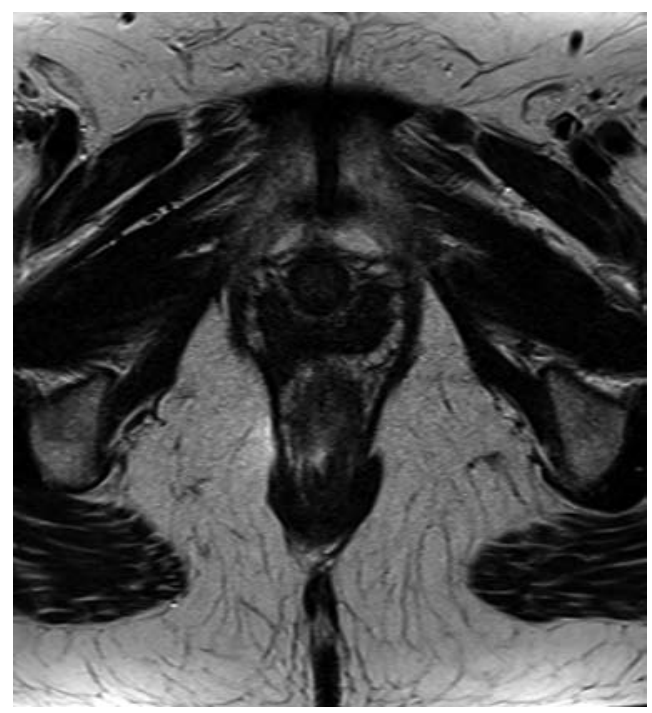

Figura 1. Corte axial de DRM ponderada en T2 TSE (turbo spin-echo) a nivel del piso pélvico (fase estática).

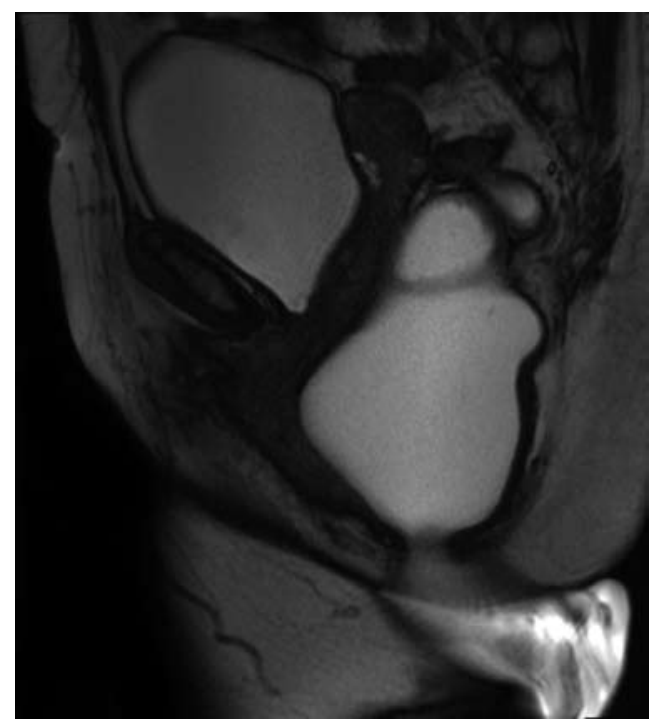

Figura 2. Corte sagital en línea media de DRM en secuencia TrueFisp (true fast imaging with steady state precession), realizado en fase dinámica durante la defecación. 


\section{Puntos de reparo anatómicos y mediciones}

Los puntos de reparo anatómicos, desde los que se realizan mediciones en relación a los compartimentos anterior, medio y posterior, son respectivamente los siguientes: piso vesical, cúpula vaginal y unión anorrectal (UAR). Esta última está definida como el punto donde se intersecta una línea que representa la pared posterior del recto distal con otra que se dispone en el eje central (cráneo-caudal) del canal anal. El ángulo formado por esta intersección es el anorrectal (AAR), que en reposo mide aproximadamente $90-100^{\circ}$ y con la contracción del esfínter anal disminuye a aproximadamente $85^{\circ}$; con las maniobras de Valsalva y defecación aumenta a $135^{\circ(8)}$.

La línea pubocoxígea inferior (LPCI) conecta el borde inferior del pubis con la última articulación coxígea. Las mediciones se realizan entre los puntos de reparo anatómicos descritos previamente y la $\mathrm{LPCl}$, en especial, durante las maniobras de Valsalva y defecación. Se considera un descenso leve, moderado o acentuado de estas estructuras (vejiga, cúpula vaginal y $U A R$ ), si el descenso es de $0-3 \mathrm{~cm}$, 3-6 $\mathrm{cm}$ o mayor a $6 \mathrm{~cm}$, respectivamente.

\section{Condiciones patológicas}

Las condiciones patológicas principales son cinco: rectocele anterior, invaginación rectal, enterocele, síndrome de descenso perineal y contracción paradójica del músculo puborrectal. Estas pueden traslaparse en grado variable, siendo frecuente la coexistencia de dos o más de ellas.

\section{Rectocele anterior:}

Es un abombamiento o extensión hacia anterior de la pared anterior del recto, secundario a soporte inadecuado por la fascia endopélvica. Es más frecuente en mujeres; puede ser leve, moderado o acentuado, si se proyecta menos de $2 \mathrm{~cm}$, entre 2 y $4 \mathrm{~cm}$, o más de $4 \mathrm{~cm}$ de la localización esperada de la pared rectal anterior normal, respectivamente (Figuras 3 y 4 ). La significación clínica del rectocele está dada por la presencia de 4 criterios: tamaño mayor a $2 \mathrm{~cm}$, retención de contraste en el rectocele, reproducibilidad de los síntomas durante el examen y, necesidad de asistencia para evacuar, como introducir el dedo en la vagina y comprimir hacia posterior para evacuar el rectocele ${ }^{(2,9)}$. Usualmente, el rectocele es secundario a pujo crónico en relación a otras causas, como por ejemplo contracción paradójica del músculo puborrectal, o bien, asociado a invaginación rectal. Es por esto que el manejo del rectocele debe ir aparejado de la solución de la causa primaria.

\section{Invaginación rectal:}

Hallazgo frecuente en la defecografía, la minoría de las veces asociada a síntomas. La invaginación o intususcepción rectal puede ser de la mucosa o com-

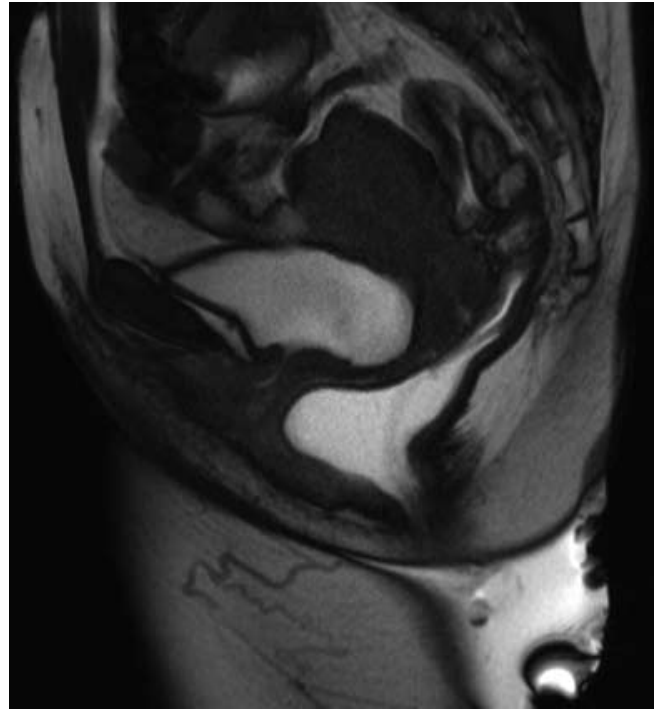

Figura 3. Corte sagital de DRM en secuencia TrueFisp. Se observa un moderado rectocele anterior que indenta el tabique rectovaginal. Además, hay un moderado descenso del piso perineal (UAR a más de $3 \mathrm{~cm}$ de la $L P C l$ ).

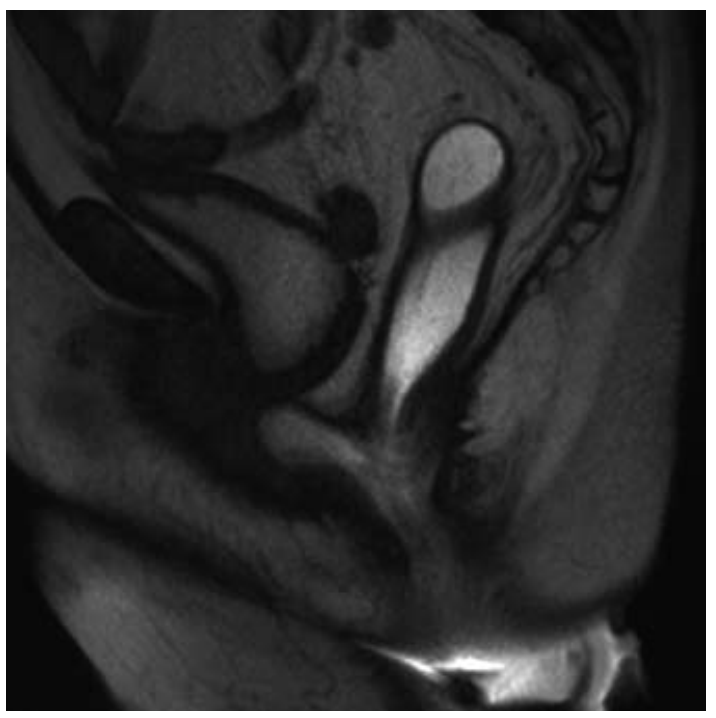

Figura 4. Corte sagital de DRM en secuencia TrueFisp. Se observa un moderado rectocele anterior asociado a invaginación intrarrectal, moderado a acentuado descenso del piso perineal, moderado enterocele (omento) y leve cistocele.

prometer la pared rectal. Consiste en un plegamiento de la pared (o mucosa), que se extiende hacia el lumen rectal determinando obstrucción parcial o completa al tracto de salida. Puede ser anterior, posterior o circunferencial y se asocia a la presencia de rectoceles anterior y/o posterior así como también a la presencia de enteroceles. Se clasifica en intra-rectal (Figura 5), intra-anal (Figura 6) y extra-anal o prolapso rectal (Figura 7). Los síntomas que se producen son: dificultad en la evacuación, descarga de mucus o sangre por el recto, dolor e incontinencia rectal (especialmente en la extra-anal o prolapso rectal). 


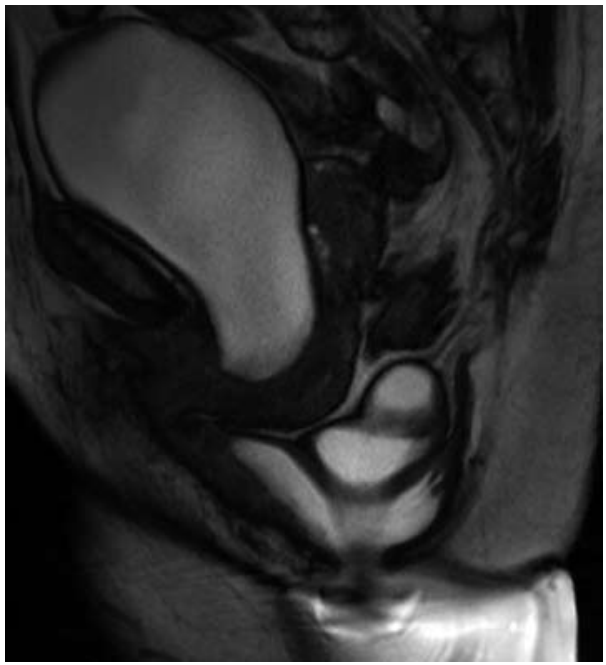

Figura 5. Corte sagital de DRM en secuencia TrueFisp. Invaginación intrarrectal concéntrica (parietal) asociada a un acentuado descenso del piso perineal (UAR a más de $6 \mathrm{~cm}$ de la $L P C \mathrm{Cl}$ ), a un moderado rectocele anterior, y a un leve a moderado cistocele y enterocele (sigmoides).

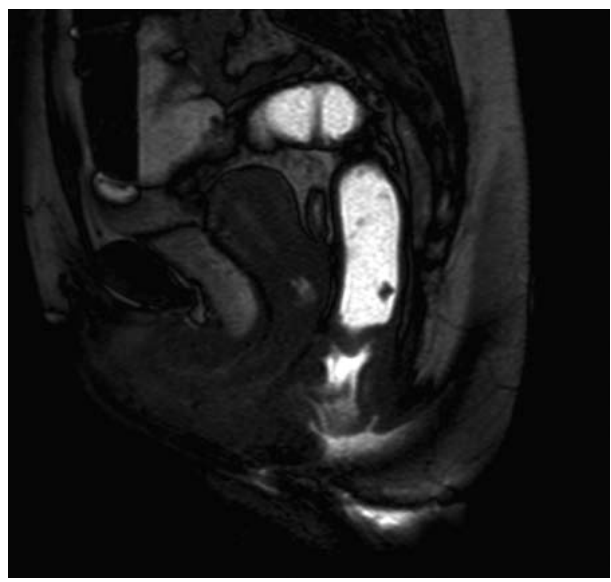

Figura 6. Corte sagital de DRM en secuencia TrueFisp. Invaginación intra-anal de mucosa rectal redundante, que determina obstrucción parcial del tracto de salida.

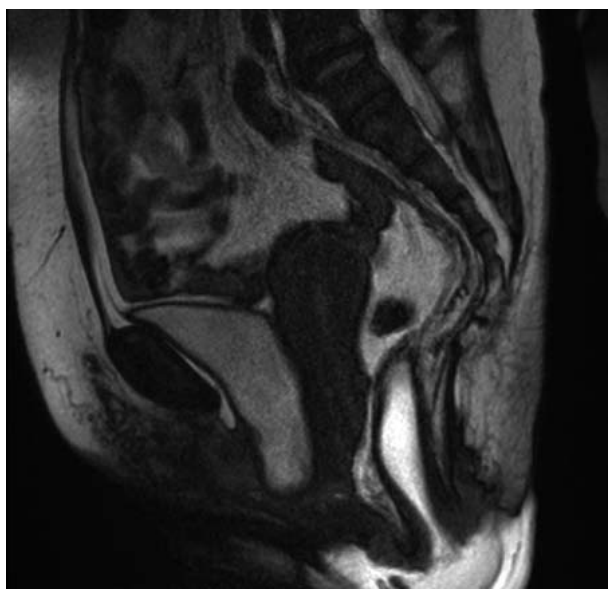

Figura 7. Corte sagital de DRM en secuencia TrueFisp. Invaginación rectal extra-anal (prolapso rectal) asociada a un acentuado enterocele, acentuado descenso del piso perineal y a un moderado cisto-histerocele.

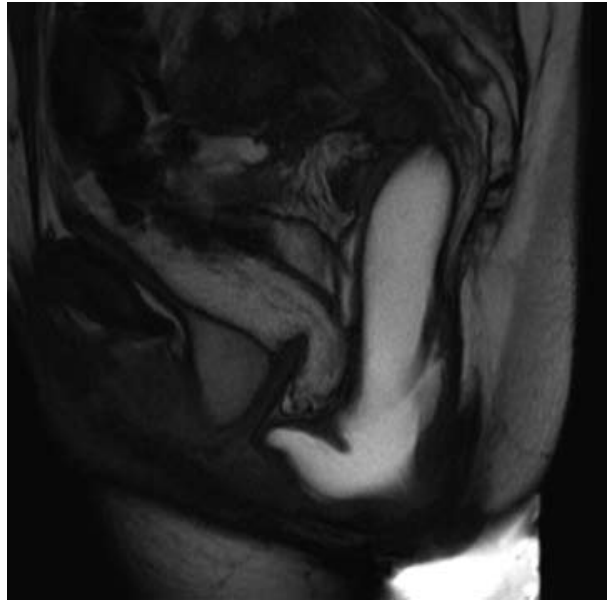

Figura 8. Corte sagital de DRM en secuencia TrueFisp. Antecedente de histerectomía. Se observa un moderado enterocele (omento y en menor grado asas de intestino delgado) que se localiza entre la vagina y el recto. Se asocia a un acentuado descenso del piso perineal, a un moderado rectocele anterior, invaginación intrarrectal y un moderado cistocele.

\section{Enterocele:}

En general existe el antecedente de histerectomía. Es la herniación de un saco peritoneal a través del fondo de saco de Douglas durante la maniobra de Valsalva o defecación, localizado anterior a la pared rectal anterior, separando la vagina del recto (tabique recto-vaginal) ${ }^{(2,10)}$ (Figura 8). Puede contener grasa omental, asas de intestino delgado o colon sigmoides. Por lo general es subdiagnosticada en el examen clínico, lo que le brinda una especial importancia a la DRM, dado que el manejo en general es quirúrgico.

El tamaño del enterocele puede ser complementario con el del rectocele cuando estos coexisten, es decir, si el rectocele es muy significativo, el enterocele es pequeño y viceversa.

En la DRM debe visualizarse la totalidad de la o las fases dinámicas, dado que el enterocele puede aparecer o acentuarse al final de la defecación (Figura 9 a y b).

\section{Sindrome de descenso perineal:}

Descrito por Parks en 1966 ${ }^{(11,12)}$, se caracteriza por la disminución patológica del tono muscular del piso pélvico con significativo descenso de las estructuras (vejiga, útero y recto), con maniobra de Valsalva y durante la defecación (Figura 10). Esto es secundario a daño del nervio pudendo, principalmente en relación a embarazos y postparto así como también al pujo crónico. Los pacientes refieren discomfort y dolor perineal, así como también sensación de defecación incompleta. 

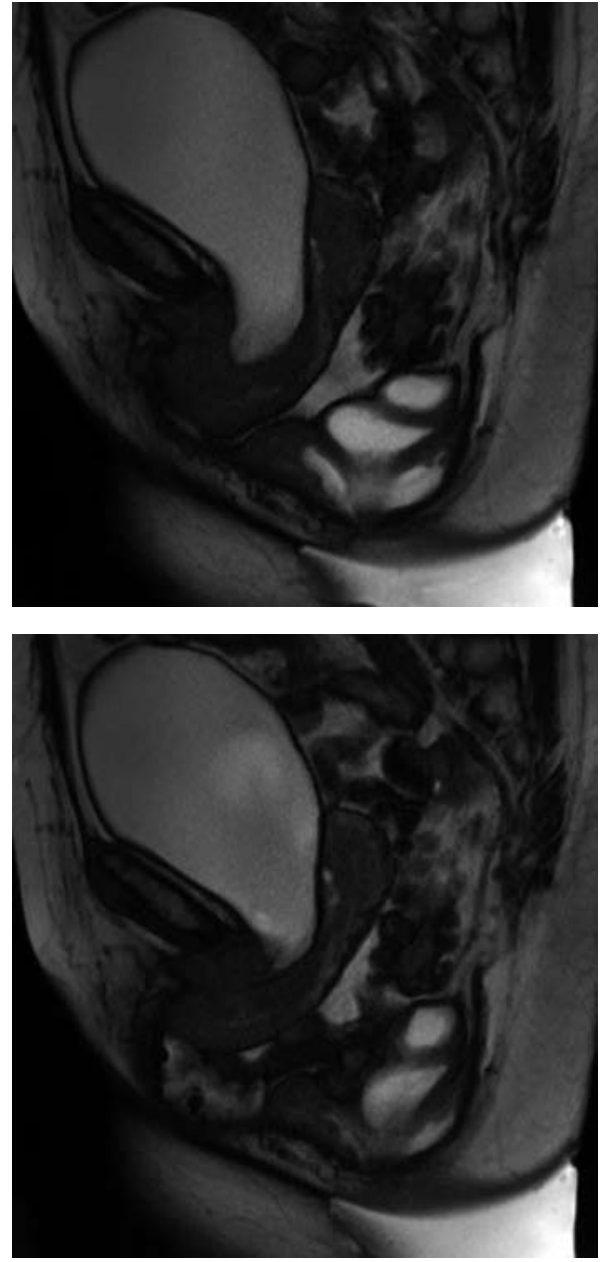

Figura 9 a $\boldsymbol{y}$ b. Cortes sagitales de DRM en secuencia TrueFisp (similar paciente que figura 5). En el inicio de la defecación se observa un leve a moderado enterocele constituído por colon sigmoides y grasa omental (a). En la fase defecatoria final se hace evidente un acentuado enterocele constituído por asas de intestino delgado que producen efecto de masa sobre la región vulvar (b).

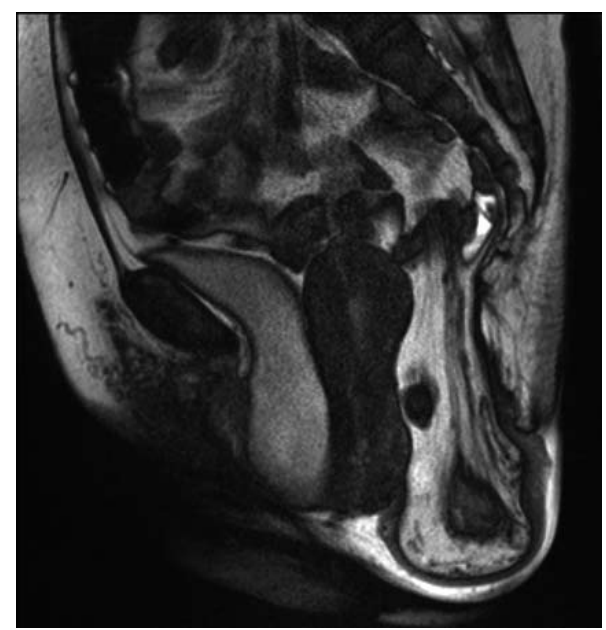

Figura 10. Corte sagital de DRM en secuencia TrueFisp. Se observa durante la fase defecatoria un más que acentuado descenso del piso perineal (UAR a más de $11 \mathrm{~cm}$ de la $L P C I$ ) asociado a un acentuado cisto-histero-enterocele.

\section{Contracción paradójica del músculo puborrectal:}

Es una relajación inadecuada del músculo puborrectal durante la defecación. Este músculo se dispone formando un asa posterior al recto, que al contraerse lo desplaza hacia anterior, disminuyendo el AAR, impidiendo la defecación. Durante la maniobra de defecación, debe relajarse aumentando este ángulo (se verticaliza). En estos pacientes, al no relajarse adecuadamente este músculo, se produce obstrucción parcial al tracto de salida. Puede asociarse a rectocele anterior y a disminución de calibre del recto (Figura 11). El tratamiento es con biofeedback ${ }^{(13)}$.

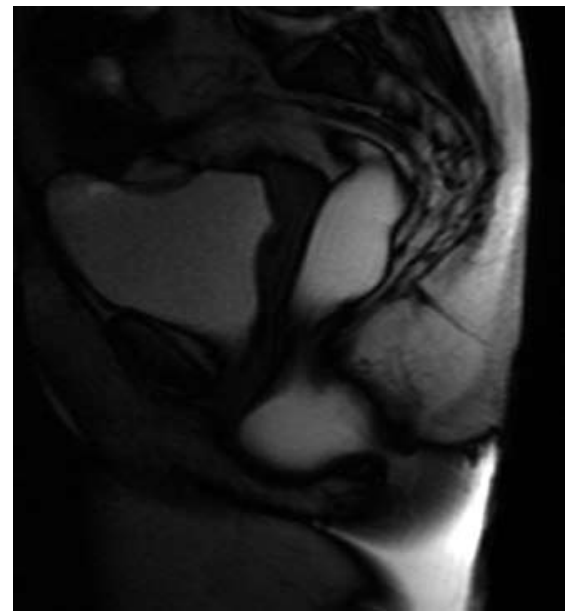

Figura 11. Corte sagital de DRM en secuencia TrueFisp. Contracción paradójica del músculo puborrectal, determinando un $A A R$ de aproximadamente $90^{\circ}$, asociado a rectocele anterior y disminución de calibre del recto, que determina obstrucción parcial del tracto de salida.

\section{Postquirúrgicas:}

Muchos pacientes han requerido más de una cirugía para intentar solucionar los problemas del piso pélvico, en parte porque el manejo de estas patologías es complejo y requiere un enfoque multidisciplinario, que incluye participación de urólogos, ginecólogos, coloproctólogos, kinesiólogos y psiquiatras, entre otros. Por otra parte, si no se realiza un estudio adecuado, hay patologías que pueden quedar subdiagnosticadas, como por ejemplo el enterocele (Figura 12). Esto reafirma el rol de las imágenes y de las reuniones clínicas, en las que se pueden determinar soluciones en conjunto. Además, la técnica quirúrgica debe ser la más apropiada (Figura 13 a y b) para la patología del paciente. 


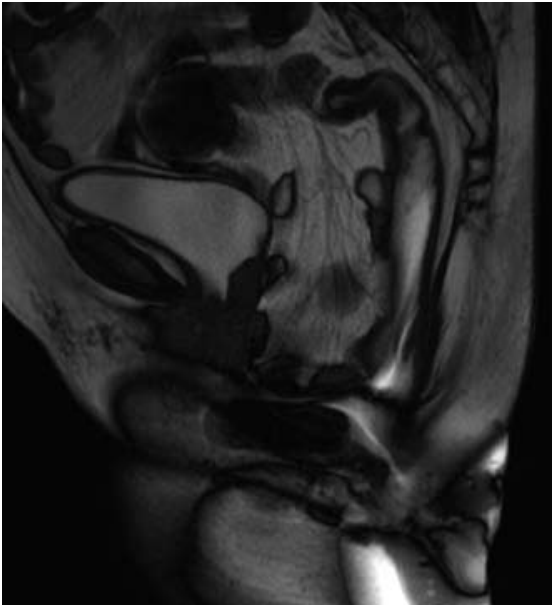

Figura 12. Corte sagital de DRM en secuencia TrueFisp. Mujer con antecedente de cirugía en piso pélvico, con malla a nivel del tabique recto-vaginal que se visualiza como artefacto, que consulta por sensación de evacuación incompleta. Se identifica un moderado enterocele que ocupa la parte superior del tabique recto-vaginal, comprimiendo el recto.
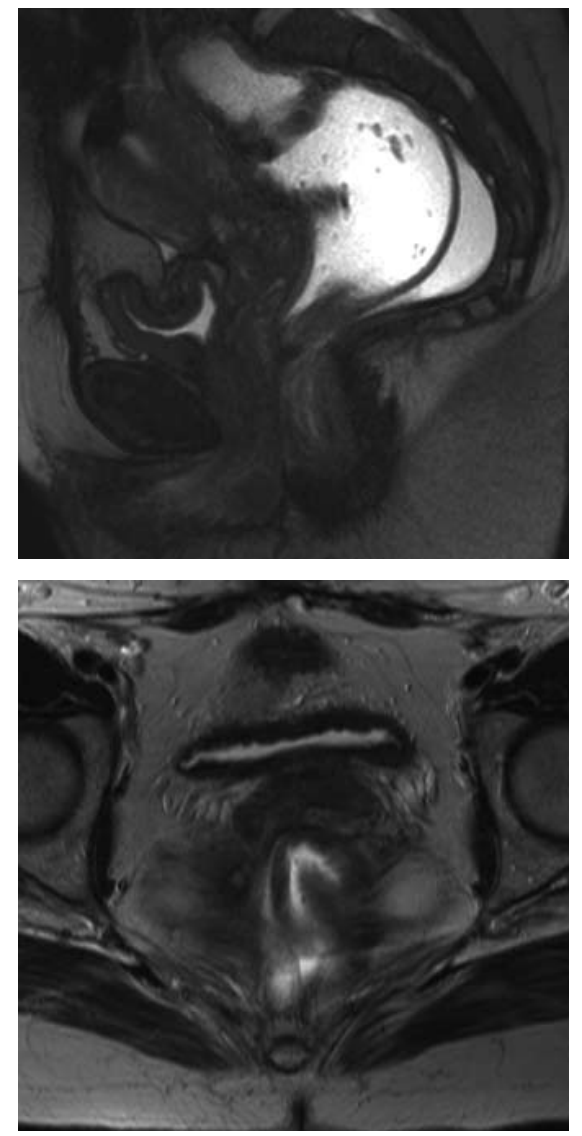

Figura 13 a y $\boldsymbol{b}$. Cortes sagital de DRM en secuencia TrueFisp (a) y axial T2 TSE (b). Mujer con antecedente de proctocolectomía total con reservorio ileal y anastomosis íleo-anal, que refiere incapacidad de defecar desde que se realizó la cirugía. Se observa una colección hiperintensa en situación presacra. Durante la fase defecatoria, la paciente no logra defecar (a). En corte axial T2 (b), se observa una torsión del reservorio distal en $180^{\circ}$, adyacente a la anastomosis.

\section{Conclusión}

La DRM es una modalidad rápida y de alto rendimiento para la evaluación anatómica y funcional del piso pélvico. Evalúa los tres compartimentos, visualiza los tejidos blandos perirrectales, es menos invasiva que los métodos convencionales y no utiliza radiación ionizante. Por estas razones, se recomienda como estudio de primera línea para la evaluación de las patologías del piso pélvico.

\section{Bibliografía}

1. Drossman DA, Li Z, Andruzzi E, Temple RD, Talley NJ, Thompson WG et al. U.S. householder survey of functional gastrointestinal disorders: prevalence, sociodemography, and health impact. Dig Dis Sci 1993; 38: 1569-1580.

2. Roos $\mathrm{J}$, Weishaupt $\mathrm{D}$, Wildermuth $\mathrm{S}$, Willmann $\mathrm{J}$, Marincek B, Hilfiker P. Experience of 4 years with open MR Defecography: Pictorial review of anorectal anatomy and disease. RadioGraphics 2002; 22: 817832.

3. Cofré $P$, Germain F, Medina L, Orellana $H$, Suárez $\mathrm{J}$, Vergara T. Manejo de la constipación crónica del adulto: actualización. Rev méd Chile 2008; 136: 507516.

4. Wallden L. Defecation block in cases of deep rectogenital pouch. Acta Chir Scand 1952; suppl 165: 1-121.

5. Colaiacomo MC, Masselli G, Polettini E, Lanciotti S, Casciani E, Bertini L, et al. Dynamic MR Imaging of the Pelvic Floor: a Pictorial Review. Radiographics 2009 29: e35; doi: 10.1148/rg. e35

6. Bertschinger KM, Hetzer FH, Roos JE, Treiber K, Marincek B, Hilfiker PR. Dynamic MR imaging of the pelvic floor performed with patient sitting in an openmagnet unit versus with patient supine in a closedmagnet unit. Radiology 2002; 223(2): 501-508.

7. Anthuber C, Baron A, Kohz P, Reiser M. Dynamic MR colpocystorectography assessing pelvic-floor descent. Eur Radiol 1997; 7: 1309-1317.

8. Schoenenberger AW, Debatin JF, Guldenschuh I, Hany TF, Steiner P, Krestin GP. Dynamic MR defecography with a superconducting, open-configuration MR system. Radiology 1998; 206: 641-646.

9. Pfeifer J, Oliveira L, Park UC, González A, Agachan F, Wexner SD. Are interpretations of video defecographies reliable and reproducible? Int $\mathrm{J}$ Colorectal Dis 1997; 12: 67-72.

10. Altringer WE, Saclarides TJ, Domínguez JM, Brubaker LT, Smith CS. Four-contrast defecogra-phy: pelvic "floor-oscopy". Dis Colon Rectum 1995; 38: 695699.

11. Pinedo G. Constipación crónica y cirugía. Rev Chil Cir 2007; 59: 305-310.

12. Parks AG, Porter NH, Hardcastle J. The syndrome of the descending perineum. Proc R Soc Med 1966; 59: 477-482.

13. Bassotti G, Chistolini F, Sietchiping-Nzepa F, de Roberto G, Morelli A, Chiarioni G. Biofeedback for pelvic floor dysfunction in constipation. BMJ 2004; 328: 393-396. 